\title{
Editorial
}

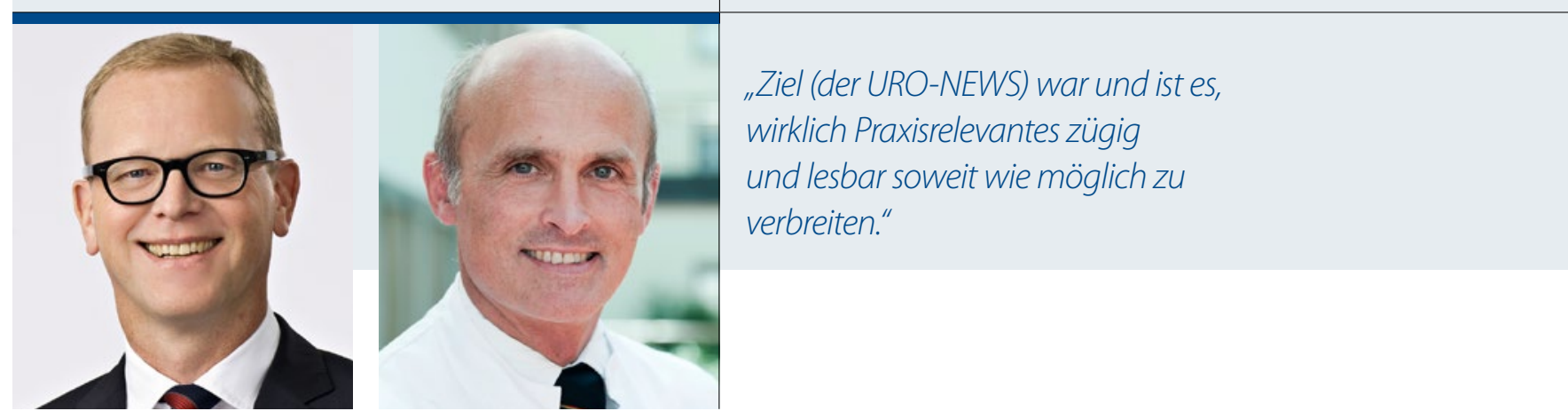

Prof. Dr. med. Elmar W. Gerharz Prof. Dr. med. Andreas J. Gross

Urologie an der Paulskirche, Klinik für Urologie, Asklepios Klinik

UROGATE, Frankfurt am Main Barmbeck, Hamburg

\section{Bat all passiert!}

$\mathrm{D}$ ie deutsche Hochsprache schöpfe ihre ewige Kraft aus ihren Mundarten, schreibt sinngemäß der Bismarck-Biograf Ludwig Reiners in seiner zeitlos lesenswerten Stilfibel „Der sichere Weg zum guten Deutsch“. Die aus dem Westerwälder Heimatort eines der Chefredakteure übernommene Überschrift unseres Neujahrs-Editorials bedeutet „Was alles passiert" und beschreibt neben dem Anspruch, Zeitgeschehen zu protokollieren auch das fast fatalistische Erstaunen angesichts desselben.

Über einen Mangel an Erstaunlichem können wir uns im Rückblick auf 2016 wahrlich nicht beklagen. Der erste URO-NEWS-Jahrgang in unserer Verantwortung fiel in eine Zeit, in der die Alte und die Neue Welt in ihren Grundfesten erschüttert wurden. Einige der Entwicklungen haben wir in unserem Einstands-Editorial im Februar letzten Jahres adressiert, nicht ahnend, dass es schlimmer kommen würde. Die sonst so kühl kalkulierenden Engländer erweisen sich als so chaotisch wie die Frisur von Boris Johnson, in Amerika formiert sich ein skurriles Kabinett der Milliardäre und Wrestling-Queens, und in der Hölle von Aleppo blickt die Menschheit wieder einmal in die hässliche Fratze der dunklen Seite der Macht.

Ganz im Gegensatz zu diesen verstörenden Zynismen fällt unsere persönliche URO-NEWS-Bilanz durchweg positiv aus. Ziel war und ist es, wirklich Praxisrelevantes zügig und lesbar soweit wie möglich zu verbreiten. Das scheint vor allem mit der Serie zur Mikrobiologie, mehreren exzellenten Artikeln zur multiparametrischen MRT, einem viel beachteten Text zum Vergleich verschiedener Fusionsbiopsiesysteme und Heften zu Urologie im Kindes- und hohen Alter und den urologischen Tumoren gelungen. Der in der elektronischen Version unserer Zeitschrift am häufigsten aufgerufene Aufsatz stammt übrigens aus der Feder eines Nephrologen („Wie viel Wasser braucht der Mensch zum Leben?"). Natürlich freuen wir uns besonders über die überwiegend wohlwol- lende Resonanz auf die Kongressausgabe FUTURONEWS mit ihren hochkarätigen Autoren. In der Rubrik „Wunder Punkt" haben wir in teilweise polemischer Engführung Texte zur Geriatrie, PREFERE und irreversiblen Elektroporation veröffentlicht und uns damit klar zu der identitätsstiftenden URONEWS-Tradition unabhängiger und kritischer Berichterstattung bekannt. Das soll auch so bleiben. Immerhin hat das kinderurologische Heft zu einer sehr lebhaften Diskussion auf höchster fachgesellschaftlicher Ebene geführt. Ob die geplante gemeinsame Weiterbildungsordnung mit den Kinderchirurgen jemals Realität wird, bleibt abzuwarten. Ein URO-Kult zu Bob Dylan darf durchaus als Prodrom des Literaturnobelpreises angenommen werden, und auch das Wort des Jahres 2016 - postfaktisch - haben wir kurz vor der Bekanntgabe bereits ironisierend verarbeitet.

In dieser Ausgabe dreht sich wie bereits im Dezember alles um das Urothelkarzinom. Themen und Autoren wurden von Frau Retz und ihrem Münchener Team vorgeschlagen, das eine Executive Summary der S3-Leitlinie zur zweithäufigsten urologischen Tumorerkrankung beigesteuert hat. Bei der Gelegenheit darf dankbar und mit größtem Respekt erwähnt werden, was für eine immense, unentgeltliche („Für die Ehre") Arbeit in der Erstellung von Behandlungsleitlinien steckt! Ähnlich dankbar sind wir, dass wir mit Christian Doehn aus der Buddenbrook-Stadt Lübeck einen weiteren erfahrenen, im universitären Stahlgewitter ertüchtigten niedergelassenen Urologen als Aktivposten im Beirat begrüßen durften. Nehmen wir diese Personalie als positiv prädiktiven Wert für die weitere Entwicklung von URO-NEWS. Schließlich wird die Zeitschrift mit den unverwechselbaren Cover-Cartoons in diesem Jahr 20 Jahre alt.

Here's to you, URO-NEWS!
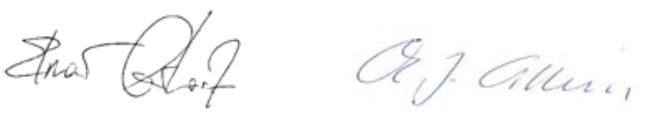